\title{
Application of two novel electrical restitution based ECG markers of ventricular arrhythmia to patients with non-ischemic cardiomyopathy
}

\author{
William Nicolson ${ }^{1}$, Matthew Smith ${ }^{1}$, Zakariyya Vali ${ }^{1}$, Nilesh Samani ${ }^{1}$, and G. André $\mathrm{Ng}^{2}$ \\ ${ }^{1}$ University of Leicester \\ ${ }^{2}$ Glenfield Hospital
}

May 27, 2020

\begin{abstract}
Introduction: Sudden Cardiac Death (SCD) risk assessment is limited, particularly in patients with non-ischemic cardiomyopathies. This is the first application, in patients with non-ischemic cardiomyopathies, of two novel risk markers, Regional Restitution Instability Index (R2I2) and Peak Electrocardiogram Restitution Slope (PERS), which have been shown to be predictive of ventricular arrhythmias (VA) or death in ischemic cardiomyopathy patients. Methods and Results: Blinded retrospective study of 50 patients: 33 dilated cardiomyopathy and 17 other; undergoing electrophysiological study (EPS) for SCD risk stratification, and 29 controls with structurally normal hearts undergoing EPS. R2I2 was calculated from an EPS using ECG surrogates for action potential duration and diastolic interval. Cut-offs for high and low R2I2/PERS were predefined. R2I2 was significantly higher in study than control patients $(0.99 \pm 0.05$ vs. $0.63 \pm 0.04,<0.001)$. PERS showed a trend to higher values in the study group (1.18[0.63] vs. 1.09[0.54], $\mathrm{p}=0.07)$. During median follow up of 5.6 years [IQR 1.9 years] 9 study patients reached the endpoint of ventricular arrhythmia(VA)/death. Patients who experienced VA/death showed trends to higher mean R2I2 (1.14 $\pm 0.07 v s .0 .95 \pm 0.05, \mathrm{p}=0.12)$ and PERS (1.46[0.49] vs. 1.13[0.62], $\mathrm{p}=0.22)$. A Cox proportional hazards model using grouped markers: R2I2<1.03+PERS $<1.21$ / either R2I2[?]1.03 or PERS[?]1.21 / R2I2[?]1.03+PERS[?]1.21; significantly predicted VA/death $(\mathrm{p}=0.02)$ with a hazard ratio per positive component of $3.2(95 \%$ confidence interval 1.2 to 8.8$)$. Conclusion: R2I2[?]1.03+PERS[?]1.21 predict VA/death in patients with non-ischemic cardiomyopathies. R2I2[?]1.03+PERS[?]1.21 have the potential to play an important role in SCD risk stratification in non-ischemic cardiomyopathies but their validity should be confirmed in a larger study.
\end{abstract}

\section{INTRODUCTION}

Sudden cardiac death (SCD) is a major cause of preventable mortality, estimated to be responsible for over 6 million deaths per year worldwide. ${ }^{1}\{\{86$ Myerburg, M Risk stratification of patients for implantable cardioverter defibrillator (ICD) therapy has major limitations, particularly for patients at risk due to a non-ischemic cardiomyopathy, who make up $20 \%$ of SCD cases. $^{2}$ Current electrophysiological (EP) and non-invasive predictive methods for the identification of non-ischemic patients at high risk for SCD have been shown to be inaccurate. ${ }^{3}$ The recent DANISH study has further highlighted the need for better risk stratification in this patient group. ${ }^{4}$

Action potential duration (APD) restitution describes the property that myocardial APD is determined by the length of the preceding diastolic interval (DI). In silico models suggest that abnormal APD restitution is arrhythmogenic through two mechanisms: increased heterogeneity of APD restitution and increased APD restitution slope steepness. ${ }^{5-8}$ Body surface electrocardiogram (ECG) surrogates for APD and DI have been used to successfully plot APD restitution in dog and swine models and have been shown to correlate with 
epicardial unipolar electrograms. ${ }^{9,10}$ Our research group has developed two novel, independent, high resolution 12-lead ECG measures of APD restitution pathology: Regional Restitution Instability Index (R2I2, a measure of APD restitution heterogeneity) and Peak Electrocardiogram Restitution Slope (PERS, a measure of APD restitution slope). ${ }^{11}$ R2I2 and PERS have been shown to be predictive of ventricular arrhythmia (VA)/death in three cohorts of patients with ischemic cardiomyopathy, and to be independent of standard risk markers. ${ }^{11,12}$ The aim of this study was to explore the application of R2I2 and PERS as risk stratification markers in a cohort of patients with non-ischemic cardiomyopathy.

\section{METHODS}

Ethical approval for this study was sought but deemed unnecessary by the Leicestershire Research Ethics Committee, and the study protocol was approved by the Research and Development Office of the University Hospitals of Leicester National Health Service Trust. The study group was identified by screening the University Hospitals of Leicester NHS Trust Cardiology Department's audit databases for patients who had undergone an electrophysiology study (EPS) with programmed electrical stimulation as part of clinical risk stratification for ICD implantation between $1^{\text {st }}$ January 2004 and $31^{\text {st }}$ July 2009 . Patients were excluded if they had a history of ischemic heart disease or Brugada syndrome (these patients are being investigated in a separate study). This identified 53 suitable patients, 3 of whom were later excluded because of insufficient data due to breakthrough / non-captured beats, providing a final cohort of 50 patients: 33 with dilated cardiomyopathy (DCM) and 17 with 'other conditions' - 5 with myotonic dystrophy, 5 with non-compaction cardiomyopathy, 3 with arrhythmogenic right ventricular dysplasia, 1 cardiac sarcoidosis, 1 hypertrophic cardiomyopathy, 1 cardiac amyloidosis and 1 apparently normal heart ventricular tachycardia.

A 30 patient control group was selected from patients undergoing an EPS for clinical management of supraventricular tachycardia in 2010. Controls were excluded prior to analysis if they had: insufficient surface ECG data recorded, an abnormal echocardiogram, family history of SCD or diabetes mellitus (because of potential for associated autonomic dysfunction and silent ischemic heart disease). During data analysis one control patient was excluded because the quadripolar catheter became displaced from the right ventricular apex during the study. All control subjects had normal left ventricular function as judged by visual assessment of $2 \mathrm{D}$ transthoracic echocardiograms. Endpoints were assigned by an independent three member committee with access to clinical records.

The EP studies for both cohorts of patients were conducted according to standard departmental protocol. Subjects were fasted and antiarrhythmic drugs withheld 4-5 half-lives prior to the procedure. The procedure was performed under minimal sedation. Catheters were positioned as was appropriate for the clinical procedure, and the study pacing protocol delivered through a $6 \mathrm{~F}$ quadripolar catheter advanced via femoral transvenous access to the right ventricular apex. Standard 12-lead ECGs were recorded using Labsystem Pro (BARD, Lowell) at a $1 \mathrm{kHz}$ sampling rate with a high pass filter set at $0.01 \mathrm{~Hz}$ and a low pass filter set to 50 Hz. The EPS protocol consisted of a single extrastimulus programmed ventricular stimulation with 8 or 10 beat trains at drive cycle lengths (DCL)s of $600 \mathrm{~ms} / 400 \mathrm{~ms}$; reduced as necessary due to breakthrough beats. The extrastimulus was started at $500 \mathrm{~ms}$ or $360 \mathrm{~ms}$ followed by decrements of $20 \mathrm{~ms}$ to the effective refractory period. The S1S2 coupling interval describes the interval between the last beat of the drive train and the first extrastimulus. Measurements taken from the final S1 and the S2 beats for each drive train are used to calculate the R2I2/PERS.

Calculation of R2I2 and PERS has been described in full previously. ${ }^{11}$ Surface ECG recordings taken during the study were exported from Labsystem Pro (BARD, Lowell) at 16-bit resolution. Recordings were then analysed using custom software written by WBN using MATLAB (Mathworks, Natick, MA, USA) with further work to refine the software by Madeiro et al. ${ }^{13}$ All points were manually verified and corrected where appropriate by MIS and WBN. The T wave peak (Tp) was preselected as the body surface surrogate for the end of the APD as has been previously used for R2I2 and PERS. ${ }^{12}$ The diastolic interval was represented by the period from the Tp of the last S1 beat to the QRS onset (QRSo) of the S2 beat (T wave peak to QRS onset - TpQ), while the APD was taken as the interval between the QRSo and Tp (QRS onset to T wave peak - QTp) of the S2 beat. Individual APD restitution slopes for all 12 ECG leads were 
calculated using the technique of Taggart et al. ${ }^{14}$ R2I2 was taken as the mean of the standard deviations of the difference from the mean slope for all of the leads. PERS was calculated by taking the mean restitution curve slope at each S1-S2 coupling interval across the 12 ECG leads with the maximum value defined as PERS.

It was necessary to censor out some data points according to predetermined rules: 1 . Breakthrough beat occurring within the last 3 beats of the drive train (122/929 censored), 2. Point measurement being indeterminate because of artefact, unclear morphology or baseline wander (298/10081). Points that have near or identical TpQ measurements across multiple QTp intervals produce extremely steep, non-physiological gradients. To avoid skewing of the data, gradients exceeding \pm 10 were censored from analysis $(1.6 \%$ of gradients).

Example QTp/TpQ plots for study and control patients with low and high values are shown in Figure 1. Figure 1A is a control patient with low R2I2 and PERS, all 12 ECG leads are seen to follow relatively homogenous, shallow restitution slopes. Figure 1B is a second control patient, PERS is high and R2I2 is moderately low in this patient reflecting the steep but relatively uniform course of the patient's ECG restitution slopes. Figure 1C is from a patient with non-ischemic cardiomyopathy and low R2I2 and PERS who did not reach the endpoint of VA/death; this patient's ECG restitution slopes are less homogenous than Figure 1A but contrast with Figure 1D a non-ischemic cardiomyopathy patient with high R2I2 and PERS who reached the endpoint of VA/death and whose ECG restitution slopes follow markedly contrasting paths.

The study group sample size was informed by a two-sample t-test power calculation using the Satterthwaite approximation for unequal variances and assuming that study patients would have R2I2 values in keeping with our previous retrospective study of patients with ischemic cardiomyopathy (R2I2 in VA/death group compared with No VA/death group (mean \pm SD: $1.30 \pm 0.25$ vs $1.03 \pm 0.27)$ ). ${ }^{12}$ To achieve $80 \%$ power at a $5 \%$ significance level, to show that R2I2 was significantly higher in study patients reaching the endpoint of VA/death versus those not, required 10 patients reaching endpoint. Screening of departmental electrophysiology audit databases captured all available, appropriate patients but because event rates were lower than anticipated, 9 patients reached the endpoint of VA/death over median follow up of 5.6 years [IQR 1.9 years]. Hence this study is underpowered for the primary endpoint.

The predefined cut-off for a positive R2I2 test was set at [?]1.03 (no units) and the cut-off for a positive PERS test was [?]1.21 (no units) as has been used in previous studies. ${ }^{11,12}$ Previously it has been found that the best discrimination of patients reaching an endpoint of VA/SCD was achieved using a combined R2I2+PERS marker: patients positive for both R2I2 and PERS, patients positive for either R2I2/PERS, patients negative for both R2I2 and PERS.

Parametric data are expressed as mean+- standard error of the mean (SEM) and were analysed with the Student t-test; nonparametric data are expressed as median [interquartile range (IQR)] and were analysed with the Mann-Whitney U test. Proportions were analysed using a two-sided Fisher's exact test or chisquared test as appropriate. A receiver operator characteristic curve using R2I2 and PERS singly and combined was constructed in the study cohort and the area under the curve calculated. Kaplan-Meier survival curves were constructed for patient subgroups partitioned by R2I2[?]1.03, PERS[?]1.21 and combined R2I2+PERS; comparison of cumulative endpoints was based on logarithmic transformations. Survival was recorded as time to first VA / death or the end of follow-up, which was capped at 6 years in view of the potential for arrhythmia substrate to change over time. In the study group Cox proportional hazards models were used to estimate hazard ratios for R2I2[?]1.03 and PERS[?]1.21 and to look for independence of combined R2I2+PERS from aetiology; independence from aetiology was chosen for multivariate analysis because of the preponderance of events in the 'other conditions' group. Spearman's rank correlation was used to look for correlation between parametric and non-parametric data. $\mathrm{P}<0.05$ was considered statistically significant. All analyses were performed using STATA (StataCorp LP, College Station, TX, USA).

\section{RESULTS}

The main clinical characteristics, R2I2 and PERS values, for the 50 study and 29 control subjects are 
summarised in Table 1. Controls had higher LVEF than study patients. R2I2 was significantly higher in study than in control patients both as a continuous and binary variable $(0.99+-0.05$ vs. $0.63+-0.04,<0.001)$. PERS showed a non-significant trend to higher values in the study group, particularly when used as a continuous variable $(1.18[0.63]$ vs. $1.09[0.54], \mathrm{p}=0.07)$. Moderate positive correlation was seen between R2I2 and PERS in controls (Figure 2; $\mathrm{r}=0.71, \mathrm{p}<0.01$ ). No correlation was seen between R2I2 and PERS in study patients $(\mathrm{r}=0.20, \mathrm{p}=0.17)$.

Median follow up in the study group was 5.6 years [IQR 1.9 years] during which 9 patients reached the endpoint of VA/death: $6 \mathrm{VA}$ and 5 deaths (2 patients had successful ICD therapy for VA and subsequently died). ICDs were implanted in 16/50 study patients. Three endpoints occurred in the 34 study group patients who did not have ICDs, 1 patient survived a VF arrest and 2 died. Characteristics of the study patients partitioned on the basis of the primary endpoint of VA/death are shown in Table 2. Patients who experienced VA/death showed non-significant trends to higher mean R2I2 (1.14+-0.07 vs. 0.95+-0.05, $\mathrm{p}=0.12)$ and PERS $(1.46[0.49]$ vs. 1.13[0.62], $\mathrm{p}=0.22)$ than those that did not. Combined R2I2+PERS was a significant predictor of the primary endpoint $(\mathrm{p}=0.01)$. Comparison of primary endpoint prediction in patients positive for both R2I2 and PERS with patients negative for both R2I2 and PERS gave sensitivity $100 \%$, specificity $74 \%$, positive predictive value $40 \%$ and negative predictive value $100 \%$.

Kaplan-Meier survival curves were constructed using the pre-defined R2I2 value of 1.03, the predefined PERS value of 1.21 and combined R2I2+PERS to partition patients into high and low risk groups as shown in Figure $3 .{ }^{11}$ Individually a trend to higher rates of VA/death was seen in patients positive for R2I2[?]1.03 $(\mathrm{p}=0.08)$ and PERS[?]1.21 ( $\mathrm{p}=0.06)$ compared to negative patients. In combination, patients positive for either/both R2I2[?]1.03+PERS[?]1.21 had significantly higher rates of VA/death than negative patients (7\% annual incidence vs. $0 \%$ annual incidence, $\mathrm{p}<0.05)$. A receiver operating characteristic analysis found that R2I2 significantly discriminated between those experiencing VA/death and those not during follow up (area under curve of 0.70, Figure 4). Further receiver operating characteristic analysis of PERS found a trend to discrimination of patients experiencing VA/death from those without (area under curve of 0.61) and no significant additional benefit from combination of R2I2 and PERS (area under curve of 0.71).

Cox proportional hazards analysis found trends to higher rates of VA/death in patients with R2I2[?]1.03 (hazard ratio 3.3, $\mathrm{p}=0.09$ ) and PERS[?]1.21 (hazard ratio 4.0, $\mathrm{p}=0.08$ ) compared to those with negative values. In a Cox proportional hazards model combined R2I + PERS significantly predicted VA/death $(\mathrm{p}=0.02)$ with a hazard ratio per positive component (i.e. R2I2 positive / PERS positive) of 3.2 (95\% confidence interval 1.2 to 8.8). Aetiology other than DCM compared with DCM was also associated with VA/death (hazard ratio 4.5, $\mathrm{p}=0.04$ ). In multivariate analysis using a Cox proportional hazards model of combined R2I2+PERS and aetiology, only R2I2+PERS remained independently predictive of VA/death (hazard ratio $2.7, \mathrm{p}=0.04)$.

Characteristics of the study patients partitioned on the basis of the aetiology are shown in Table 3 . There were significant differences in in age, left ventricular ejection fraction and medications usage between the cohort of patients with dilated cardiomyopathy compared with those with other diagnoses. R2I2 was higher in DCM patients versus controls $(1.00+-0.06$ vs. $0.63+-0.04, \mathrm{p}<0.001)$ and 'other conditions' versus controls $(0.96+-0.08$ vs. $0.63+-0.04, \mathrm{p}<0.001)$. PERS showed a trend to higher values in DCM patients versus controls $(1.25+-0.10$ vs. $1.06+-0.09, \mathrm{p}=0.18)$ and in 'other conditions' versus controls $(1.36+-0.14$ vs. $1.06+-0.09$, $\mathrm{p}=0.07$ ). Study aetiology subgroup analysis of VA/death showed non-significant trends to higher R2I2/PERS values in patients reaching the endpoint of $\mathrm{VA} /$ death compared with those not. A preponderance of endpoints occurred in the 'other conditions' subgroup (6/17 patients) compared to the dilated cardiomyopathy subgroup $(3 / 33$ patients, $\mathrm{p}=0.05)$.

\section{DISCUSSION}

In this paper, we report the first data for use of R2I2 and PERS as markers of VA/death in patients with nonischemic cardiomyopathy and we also report the first data for PERS values in a normal control population. As has been found previously, combining R2I2+PERS offered the greatest discrimination of endpoint in this 
study population, predicting VA/death $(\mathrm{p}=0.02)$ with a hazard ratio per positive component marker of 3.2. ${ }^{11}$ R2I2 and PERS separately show trends towards significantly higher values in patients reaching the endpoint of VA/death than those not. This replicates the findings of our previous work in ischemic cardiomyopathy using the same technique and cut-offs for R2I2/PERS analysis and the same endpoints. ${ }^{11,12}$ R2I2 and PERS appear to perform favourably compared to other surface ECG markers, such as T wave alternans, QRS fragmentation, signal averaged ECG and QRS-T angle, all of which have been shown to have a poor positive predictive value. $^{15}$

The underlying aetiology of the cohort was heterogeneous and significantly more endpoints occurred in the 'other conditions' subgroup compared to the dilated cardiomyopathy subgroup. Combined R2I2+PERS, was independent of aetiology in predicting $\mathrm{VA} /$ death $(\mathrm{p}=0.04)$ with a hazard ratio of 2.7 per positive component. This suggests that R2I2+PERS are applicable to both dilated cardiomyopathy patients and patients with 'other conditions' but larger studies are needed to confirm the relationship. In Kaplan Meier analysis (Figure 3) the rate of VA/death at 5 years in patients positive for both R2I2[?]1.03+PERS[?]1.21 was 41\%, compared with $23 \%$ for patients positive for either, and $0 \%$ for patients negative for both.

R2I2 was developed to quantify heterogeneity in APD restitution as a measure of electrical instability. In silico modelling has shown that heterogeneity of APD restitution leads to increased dispersion of depolarisation and repolarisation times leading to wavebreak and the substrate for ventricular arrhythmogenesis. ${ }^{5}$ Heterogeneity in APD restitution has been clinically demonstrated with both endocardial and epicardial approaches; using intracardiac catheters and also with an epicardial sock of electrodes in patients undergoing cardiac surgery, with significant differences seen between patients with ischemic heart disease and aortic valve disease. ${ }^{16-18}$ PERS is based on the original 'restitution hypothesis': a maximum APD restitution curve gradient $>1$ magnifies oscillations of APD/DI leading to wavebreak and ventricular fibrillation. Steep APD restitution curves have been shown in silico and in animal studies to convey increasing risk of VA. ${ }^{8,19,20}$

It is important and expected that R2I2 and PERS could be applied successfully to patients without ischemic heart disease. Differences in APD restitution heterogeneity have been demonstrated in studies in cohorts of hypertrophic cardiomyopathy, Brugada syndrome, dilated cardiomyopathy, valvular heart disease, cardiac sarcoidosis and ARVC when compared to healthy controls. ${ }^{21-23}$ The successful application to a non-ischemic population suggests that R2I2 and PERS measure a common underlying arrhythmogenic mechanism (i.e. electrical restitution) rather than, for example, the effect of infarction on ECG morphology.

R2I2 was found to be significantly higher in the study cohort compared to the controls. PERS showed a non-significant trend to higher values in the study group compared to controls. Both the DCM and the 'other conditions' subgroups also had a significantly higher mean R2I2 than controls with trends to higher PERS values. The study was underpowered to test for a difference in R2I2/PERS values in relation to VA/death in the study group aetiology subgroups but found trends to higher values. Moderate positive correlation was seen between R2I2 and PERS values in control patients. No correlation was seen between R2I2 and PERS values in study patients.

It is of interest that correlation was seen between R2I2 and PERS in the control group but not in the study group. Our previous study of R2I2 and PERS found no correlation between the two parameters in an ischemic cardiomyopathy population and this is anticipated since they are intended to measure different APD restitution properties. ${ }^{11}$ The R2I2 calculation uses mean and standard deviations; it is a staple of the APD restitution research to calculate mean and standard deviations of APD restitution slopes. However, as the gradient of a slope steepens it increases exponentially and hence the standard deviation of a steep slope is likely to be higher than that of a shallow slope. It is possible that in a control population with homogenous APD restitution this effect leads to a relationship between PERS and R2I2 while in a population with high APD restitution heterogeneity the heterogeneity effect predominates over the standard deviation effect.

This study provides proof-of-principle that clinically relevant biomarkers of electrical restitution curve heterogeneity and peak electrical restitution curve slope can be measured using the 12 lead ECG and be applied 
to a broad spectrum of patients at risk of sudden cardiac death with a range of underlying etiologies. The annual incidence of VA/death in patients with either/both R2I2[?]1.03/PERS[?]1.21 was over 7\% and all patients experiencing VA/death were positive for either R2I2/PERS/both. A current limitation to wider use of R2I2 and PERS is that their measurement requires a 5 min programmed stimulation protocol delivered by transvenous pacing at the right ventricular apex, limiting its use to patients in whom such a protocol can be instigated. Although in the majority of patients, this currently requires an invasive study, R2I2 and PERS can be investigated non-invasively in patients with ICDs or pacemakers. This is currently being tested in a large prospective multicentre trial of patients with ischaemic cardiomyopathy (ClinicalTrials.gov Identifier: NCT03022487). We are also exploring fully non-invasive methods of obtaining a spectrum of heart rates, such as exercise and chronotropic medication to see if these methods provide the same information as that from pacing. ${ }^{24}$

Amongst the limitations of this study was that the aetiology of the study group was heterogeneous containing patients with dilated cardiomyopathy and a range of other conditions. Importantly R2I2[?]1.03+PERS[?]1.21 were independent of aetiology in predicting VA/death but larger studies of homogenous populations are needed to support the application of R2I2 and PERS in specific cohorts. This study was marginally underpowered to find a difference in R2I2, reflected in the non-significant trends seen; the results fit expectations from previous work. ${ }^{11}$ Also, VA/death does not equate to SCD, the combined endpoint is heterogeneous. The study is a single-centre study, and before any clinical application, the association of the ECG markers with VA/death should be replicated in a broader multicentre clinical trial.

This study offers the first evidence to support application of two novel electrical restitution markers R2I2[?]1.03+PERS[?]1.21 in assessment of sudden cardiac death risk in patients with non-ischemic heart disease. R2I2 and PERS have the potential to play an important role in SCD risk stratification but their validity should be confirmed in a larger multi-centre study before clinical trials are undertaken.

\section{Acknowledgements}

This study is part of the research portfolio supported by the NIHR Leicester Cardiovascular Biomedical Research Unit. We thank clinical endpoint committee members: Dr Alastair J Sandilands, Dr Peter J Stafford and Dr Riyaz Somani. We thank Dr Fernando S Schlindwein for his assistance with MATLAB script development.

\section{Disclosures}

The University of Leicester has applied for patents for the Regional Restitution Instability Index and Peak Electrocardiogram Restitution Slope techniques on behalf of Dr Will Nicolson and Professor G Andre Ng.

\section{REFERENCES}

1. Mehra R. Global public health problem of sudden cardiac death. J Electrocardiol. 2007. 40:S118-S122.

2. Myerburg RJ, Interian A, Mitrani RM, Kessler KM, Castellanos A. Frequency of sudden cardiac death and profiles of risk. American Journal of Cardiology. 1997; 80:10F - 19F.

3. Grimm W, Christ M, Bach J, Muller H-H, Maisch B. Noninvasive Arrhythmia Risk Stratification in Idiopathic Dilated Cardiomyopathy. Circulation. 2003;108:2883-2891.

4. Kober L, Thune JJ, Nielsen JC, Haarbo J, Videbaek L, Korup E, Jensen G, Hildebrandt P, Steffensen FH, Bruun NE, Eiskjaer H, Brandes A, Thogersen AM, Gustafsson F, Egstrup K, Videbaek R, Hassager C, Svendsen JH, Hofsten DE, Torp-Pedersen C, Pehrson S, DANISH Investigators. Defibrillator Implantation in Patients with Nonischemic Systolic Heart Failure. N Engl J Med. 2016;375:1221-30.

5. Clayton RH, Taggart P. Regional differences in APD restitution can initiate wavebreak and re-entry in cardiac tissue: a computational study. Biomed Eng Online. 2005;4:54.

6. Cao JM, Qu Z, Kim YH, Wu TJ, Garfinkel A, Weiss JN, Karagueuzian HS, Chen PS. Spatiotemporal heterogeneity in the induction of ventricular fibrillation by rapid pacing: Importance of cardiac restitution 
properties. Circ Res. 1999;84:1318-1331.

7. Karma A. Electrical alternans and spiral wave breakup in cardiac tissue. Chaos. 1994;4:461-472.

8. Ng GA, Brack KE, Patel VH, Coote JH. Autonomic modulation of electrical restitution, alternans and ventricular fibrillation initiation in the isolated heart. Cardiovasc Res. 2007;73:750-760.

9. Fossa AA, Wisialowski T, Crimin K. QT Prolongation Modifies Dynamic Restitution and Hysteresis of the Beat-to-Beat QT-TQ Interval Relationship during Normal Sinus Rhythm under Varying States of Repolarization. Pharmacology. 2008;:498-506.

10. Jiang H, Zhao D, Cui B, Lu Z, Lu J, Chen F, Bao M. Electrical restitution determined by epicardial contact mapping and surface electrocardiogram: its role in ventricular fibrillation inducibility in swine. J Electrocardiol. 2008;41:152-9.

11. Nicolson WB, McCann GP, Smith MI, Sandilands AJ, Stafford PJ, Schlindwein FS, Samani NJ, Ng GA. Prospective evaluation of two novel ECG-based restitution biomarkers for prediction of sudden cardiac death risk in ischaemic cardiomyopathy. Heart. 2014;100:1878-1885.

12. Nicolson WB, McCann GP, Brown PD, Sandilands AJ, Stafford PJ, Schlindwein FS, Samani NJ, Ng GA. A novel surface electrocardiogram-based marker of ventricular arrhythmia risk in patients with ischemic cardiomyopathy. J Am Heart Assoc. 2012;1:e001552.

13. Madeiro JPV, Nicolson WB, Cortez PC, Marques JAL, Vazquez-Seisdedos CR, Elangovan N, Ng GA, Schlindwein FS. New approach for T-wave peak detection and T-wave end location in 12-lead paced ECG signals based on a mathematical model. Med Eng Phys. 2013;35:1105-1115.

14. Taggart P, Sutton P, Chalabi Z, Boyett MR, Simon R, Elliott D, Gill JS. Effect of adrenergic stimulation on action potential duration restitution in humans. Circulation. 2003;107:285-289.

15. Goldberger JJ, Subačius H, Patel T, Cunnane R, Kadish AH. Sudden cardiac death risk stratification in patients with nonischemic dilated cardiomyopathy. J Am Coll Cardiol. 2014;63:1879-1889.

16. Ng GA, Mantravadi R, Walker WH, Ortin WG, Choi BR, de Groat W, Salama G. Sympathetic nerve stimulation produces spatial heterogeneities of action potential restitution. Heart Rhythm. 2009;6:696-706.

17. Yue AM, Franz MR, Roberts PR, Morgan JM. Global endocardial electrical restitution in human right and left ventricles determined by noncontact mapping. J Am Coll Cardiol. 2005;46:1067-75.

18. Nash MP, Bradley CP, Paterson DJ. Imaging electrocardiographic dispersion of depolarization and repolarization during ischemia: Simultaneous body surface and epicardial mapping. Circulation. 2003;107:22572263.

19. Nolasco JB, Dahlen RW. A graphic method for the study of alternation in cardiac action potentials. JApplPhysiol. 1968;25:191-196.

20. Xie F, Qu Z, Yang J, Baher A, Weiss JN, Garfinkel A. A simulation study of the effects of cardiac anatomy in ventricular fibrillation. J Clin Invest. 2004;113:686-93.

21. Osaka T, Yokoyama E, Hasebe H, Kodama I. Effects of chronic amiodarone on the electrical restitution in the human ventricle with reference to its antiarrhythmic efficacy. J Cardiovasc Electrophysiol. 2011;22:66976.

22. Gomes J, Finlay M, Ahmed AK, Ciaccio EJ, Asimaki A, Saffitz JE, Quarta G, Nobles M, Syrris P, Chaubey S, McKenna WJ, Tinker A, Lambiase PD. Electrophysiological abnormalities precede overt structural changes in arrhythmogenic right ventricular cardiomyopathy due to mutations in desmoplakin-A combined murine and human study. Eur Heart J. 2012;33:1942-1953. 
23. Lambiase PD, Ahmed AK, Ciaccio EJ, Brugada R, Lizotte E, Chaubey S, Ben-Simon R, Chow AW, Lowe MD, McKenna WJ. High-density substrate mapping in brugada syndrome: Combined role of conduction and repolarization heterogeneities in arrhythmogenesis. Circulation. 2009;120:106-117.

24. Ng GA, Mistry A, Li X, Schlindwein FS, Nicolson WB. LifeMap: towards the development of a new technology in sudden cardiac death risk stratification for clinical use. EP Eur. 2018;:euy080-euy080.

Table 1. Main characteristics of the study and control groups.

\begin{tabular}{llll}
\hline Cohort & Control $(\mathrm{n}=29)$ & Study $(\mathrm{n}=50)$ & P Value \\
\hline Age (years) & $45.3 \pm 2.5$ & $50.6 \pm 2.2$ & 0.13 \\
Gender (male) - number (\%) & $17(59)$ & $45(90)$ & 0.21 \\
LVEF (\%) & $64.4 \pm 1.0$ & $39 \pm 2.0$ & $<0.001$ \\
QRSD & $104 \pm 3.7$ & $121 \pm 5.9$ & 0.051 \\
R2I2 & $0.63 \pm 0.04$ & $0.99 \pm 0.05$ & $<0.001$ \\
R2I2[?]1.03 - number (\%) & $0(0)$ & $19(38)$ & $<0.001$ \\
PERS & $1.09[0.54]$ & $1.18[0.63]$ & 0.07 \\
PERS[?]1.21 - number (\%) & $10(34)$ & $24(48)$ & 0.35 \\
\hline
\end{tabular}

Parametric data are expressed as mean \pm SEM, non-parametric data are expressed as median [IQR]. Abbreviations: QRSD QRS duration, LVEF left ventricular ejection fraction, R2I2 Regional Restitution Instability Index, PERS Peak Electrocardiogram Restitution Slope.

Table 2. Study group patient characteristics split by primary endpoint.

\begin{tabular}{llll}
\hline Variable & No VA/death $(\mathrm{n}=41)$ & VA/death $(\mathrm{n}=9)$ & $\mathrm{p}$ \\
\hline Age (years) & $51 \pm 2.4$ & $47 \pm 5.4$ & 0.49 \\
Gender (male) - no.(\%) & $31(76)$ & $6(67)$ & 0.58 \\
Etiology - DCM - no.(\%) & $30(73)$ & $3(33)$ & 0.02 \\
QRS duration (ms) & $113 \pm 5.6$ & $152 \pm 18$ & 0.01 \\
NYHA Class I - no.(\%) & $20(49)$ & $1(13)$ & \\
II - no.(\%) & $17(41)$ & $4(50)$ & 0.03 \\
III - no.(\%) & $4(10)$ & $2(13)$ & \\
IV - no.(\%) & $0(0)$ & $1(13)$ & \\
LVEF (\%) & $39 \pm 2.2$ & $39 \pm 4.7$ & 0.91 \\
Diabetes Mellitus - no.(\%) & $2(5)$ & $0(0)$ & 0.50 \\
Atrial fibrillation - no.(\%) & $12(29)$ & $3(33)$ & 0.81 \\
Syncope - no.(\%) & $14(34)$ & $3(33)$ & 0.96 \\
Amiodarone - no.(\%) & $3(7)$ & $2(22)$ & 0.18 \\
Beta blocker - no.(\%) & $23(56)$ & $6(67)$ & 0.56 \\
ACE/ARB - no.(\%) & $26(63)$ & $7(78)$ & 0.41 \\
Aldosterone antagonist - no.(\%) & $7(17)$ & $0(0)$ & 0.18 \\
R2I2 & $0.95 \pm 0.05$ & $1.14 \pm 0.07$ & 0.12 \\
R2I2[?]1.03 - no.(\%) & $13(32)$ & $6(67)$ & 0.07 \\
PERS & $1.13[0.62]$ & $1.46[0.49]$ & 0.22 \\
PERS[?]1.21 - no.(\%) & $17(41)$ & $7(78)$ & 0.07 \\
Negative R2I2+PERS - no.(\%) & $17(41)$ & $0(0)$ & \\
Either R2I2/PERS - no.(\%) & $18(44)$ & $5(56)$ & 0.01 \\
Positive R2I2+PERS - no.(\%) & $6(15)$ & $4(44)$ & \\
Follow up time (years) & $5.67[1.0]$ & $3.67[3.2]$ & 0.16 \\
\hline
\end{tabular}


Parametric data are expressed as mean \pm standard error mean. (Abbreviations: VA ventricular arrhythmia, DCM Dilated Cardiomyopathy, NYHA New York Heart Association, LVEF left ventricular ejection fraction, ACE/ARB angiotensin converting enzyme inhibitor / angiotensin II receptor blocker, R2I2 regional restitution instability index, PERS peak ECG restitution slope). *NYHA data not available for 1 patient.

Table 3. Study group patient characteristics split by etiology.

\begin{tabular}{llll}
\hline Variable & DCM $(\mathrm{n}=33)$ & Other $(\mathrm{n}=17)$ & $\mathrm{p}$ \\
\hline Age (years) & $54 \pm 2.5$ & $44 \pm 3.9$ & 0.03 \\
Gender (male) - no.(\%) & $26(79)$ & $11(65)$ & 0.28 \\
QRS duration (ms) & $123 \pm 7.3$ & $117 \pm 10$ & 0.61 \\
NYHA Class I - no.(\%) & $13(41)$ & $8(47)$ & \\
II - no.(\%) & $15(47)$ & $6(35)$ & 0.51 \\
III - no.(\%) & $4(13)$ & $2(12)$ & \\
IV - no.(\%) & $0(0)$ & $1(6)$ & \\
LVEF (\%) & $33 \pm 1.8$ & $51 \pm 3.1$ & $<0.001$ \\
Diabetes Mellitus - no.(\%) & $2(6)$ & $0(0)$ & 0.30 \\
Atrial fibrillation - no.(\%) & $11(33)$ & $4(24)$ & 0.47 \\
Syncope - no.(\%) & $11(33)$ & $6(35)$ & 0.89 \\
Amiodarone - no.(\%) & $3(9)$ & $2(12)$ & 0.77 \\
Beta blocker - no.(\%) & $22(67)$ & $7(41)$ & 0.08 \\
ACE/ARB - no.(\%) & $26(79)$ & $7(41)$ & 0.008 \\
Aldosterone antagonist - no.(\%) & $6(18)$ & $1(6)$ & 0.24 \\
R2I2 & $1.00 \pm 0.06$ & $0.96 \pm 0.08$ & 0.67 \\
R2I2[?]1.03 - no.(\%) & $13(39)$ & $6(35)$ & 1.00 \\
PERS & $1.13[0.58]$ & $1.39[0.93]$ & 0.20 \\
PERS[?]1.21 - no.(\%) & $14(42)$ & $10(59)$ & 0.37 \\
Negative R2I2+PERS - no.(\%) & $11(33)$ & $6(35)$ & \\
Either R2I2/PERS - no.(\%) & $17(52)$ & $6(35)$ & 0.41 \\
Positive R2I2+PERS - no.(\%) & $5(15)$ & $5(30)$ & \\
Follow up time (years) & $5.80[1.0]$ & $5.05[2.2]$ & 0.22 \\
\hline
\end{tabular}

Parametric data are expressed as mean \pm standard error mean. (Abbreviations: VA ventricular arrhythmia, DCM Dilated Cardiomyopathy, NYHA New York Heart Association, LVEF left ventricular ejection fraction, ACE/ARB angiotensin converting enzyme inhibitor / angiotensin II receptor blocker, R2I2 regional restitution instability index, PERS peak ECG restitution slope). ${ }^{*}$ NYHA data not available for 1 patient.

\section{FIGURE LEGENDS}

Figure 1. Example QTp/TpQ plots for 4 patients .

A. Control patient with low R2I2 and Low PERS. B. Control patient with low R2I2 and high PERS. C. Nonischemic cardiomyopathy (N-ICM) patient with low R2I2 and low PERS. D. Non-ischemic cardiomyopathy patient with high R2I2 and high PERS.

For each example patient the 12 ECG leads have separate lines colour coded by ECG region. The lines are drawn point-to-point rather than as gradients to allow differentiation of the ECG leads (see online supplementary file for a gradient plot example). R2I2 is higher in the patients whose ECG leads follow dissonant QTp/TpQ paths. PERS reflects the steepest restitution curve gradient taken as a mean for all 12 ECG leads; moderately high PERS values may be seen in control patients.

Figure 2. Plot of Regional Restitution Instability Index (R2I2) against Peak Electrocardiogram Restitution Slope (PERS). 
Lines are drawn at the preselected cut-off values for R2I2 and PERS. Spearman rank correlation analysis shows correlation between R2I2 and PERS in controls $(\mathrm{r}=0.71, \mathrm{p}<0.01)$, but not in non-ischemic cardiomyopathy patients $(\mathrm{r}=0.20, \mathrm{p}=0.17)$.

Figure 3. Kaplan-Meier curve for VA/death

Patients partitioned by: R2I2<1.03 and PERS $<1.21$ / either R2I2[?]1.03 or PERS[?]1.21 / both R2I2[?]1.03 and PERS[?]1.21 ( $<<0.05, \log$ rank $)$.

Figure 4. Receiver operating characteristic curve for Regional Restitution Instability Index (R2I2), Peak Electrocardiogram Restitution Slope (PERS) and both parameters combined .

$\mathrm{VA} /$ death versus event free survival. (Area under curve: PERS $=0.61, \mathrm{R} 2 \mathrm{I} 2=0.70, \mathrm{R} 2 \mathrm{I} 2+\mathrm{PERS}=0.71$ ).

Figure 1. Example QTp/TpQ plots for 4 patients .
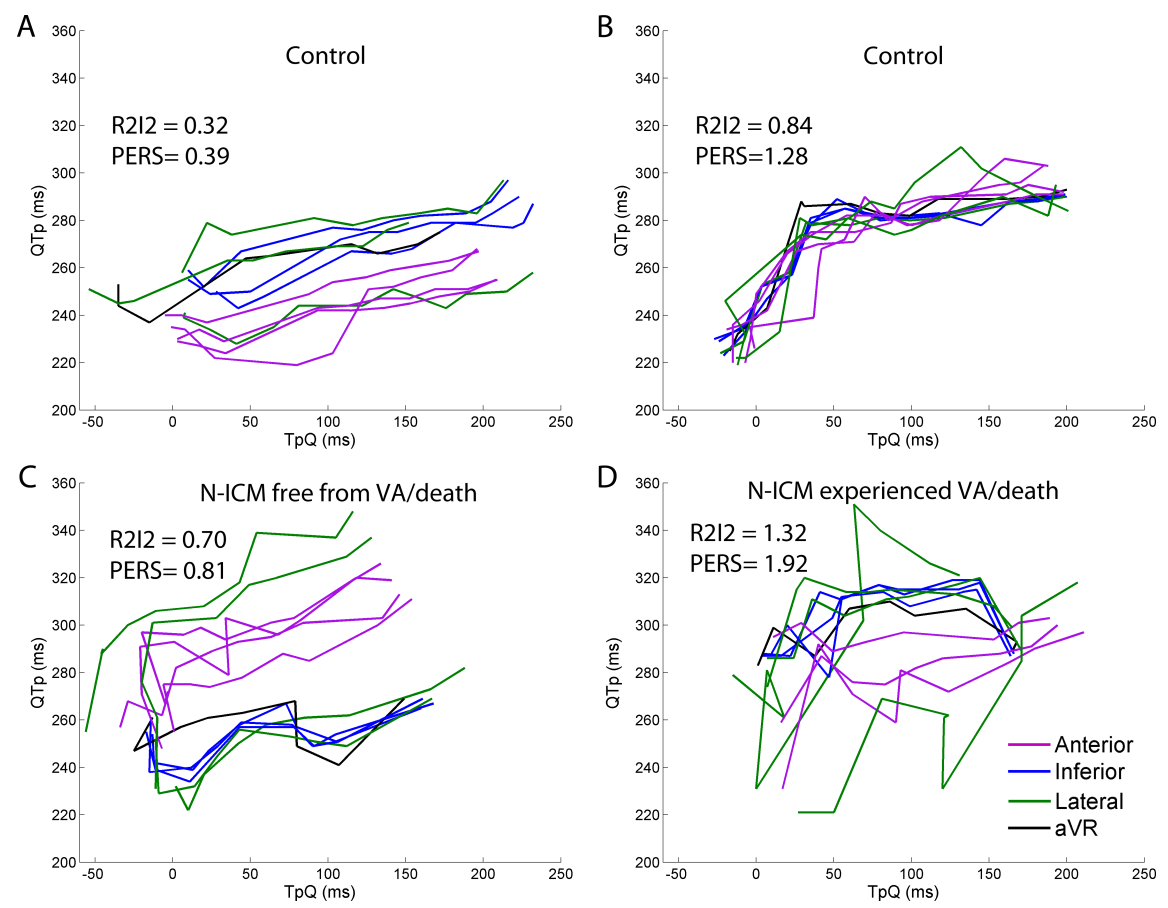

Figure 2. Plot of Regional Restitution Instability Index (R2I2) against Peak Electrocardiogram Restitution Slope (PERS). 


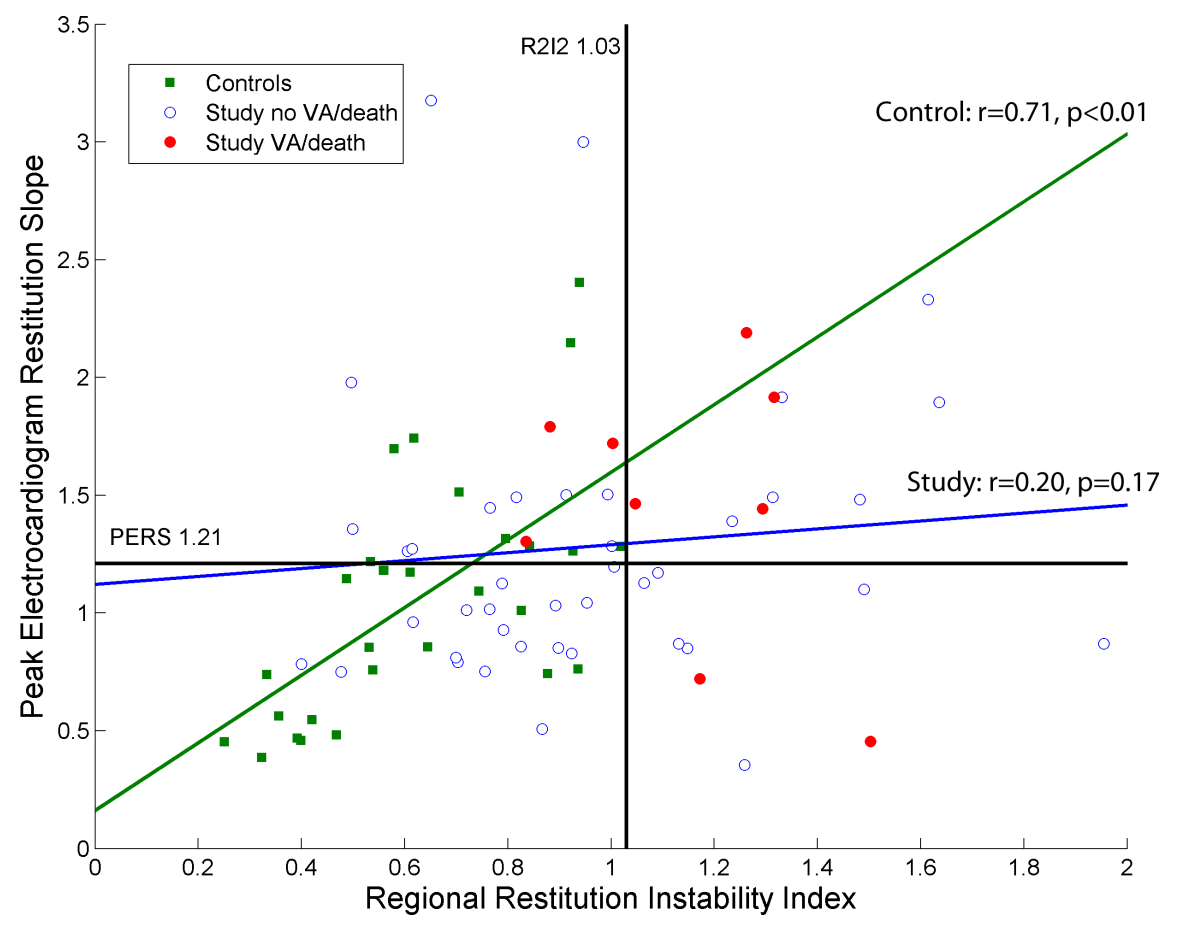

Figure 3. Kaplan-Meier curve for VA/death

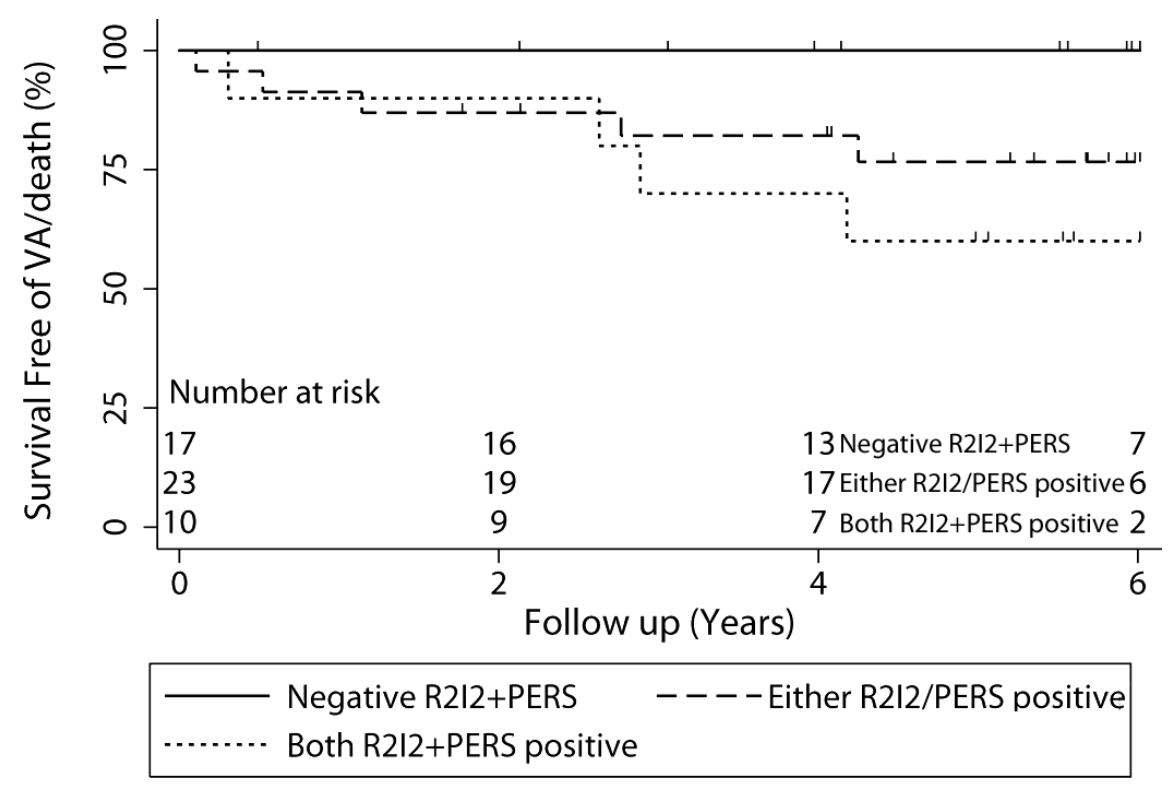

Figure 4. Receiver operating characteristic curve for Regional Restitution Instability Index (R2I2), Peak Electrocardiogram Restitution Slope (PERS) and both parameters combined . 


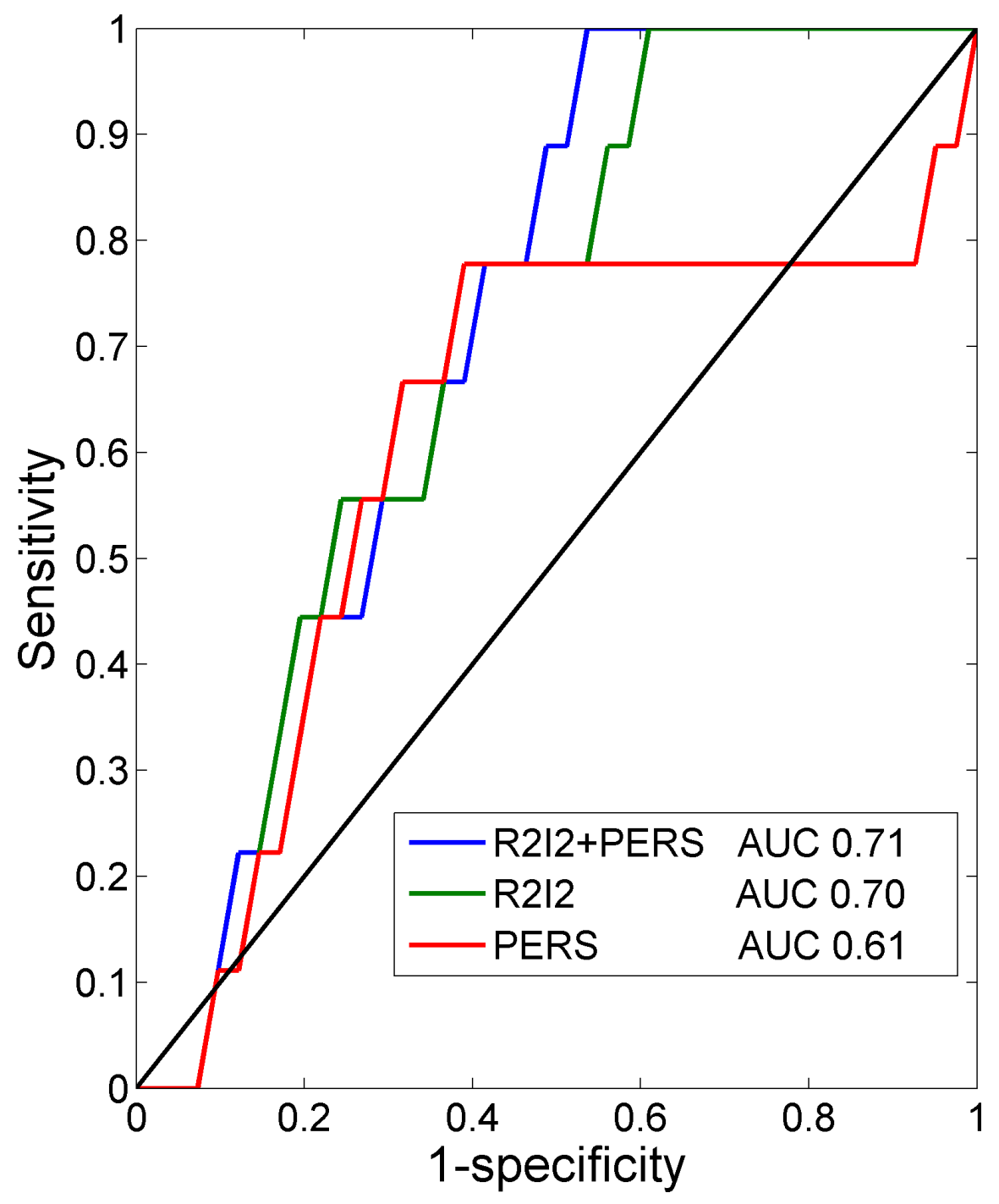

